\title{
Development and validation of the RAND Asthma Control Measure
}

\author{
Marielena Lara, Maria Orlando Edelen, Nicole K. Eberhart, Brian D. Stucky and \\ Cathy D. Sherbourne \\ Affiliation: \\ The RAND Corporation, Santa Monica, CA, USA.
}

\section{Correspondence:}

Marielena Lara, The RAND Corporation, 1776 Main Street, PO Box 2138, Santa Monica, CA 90407-2138, USA.

E-mail: laradrand.org

ABSTRACT Patient-based measures for asthma control are important in assessing the worldwide impact of this highly prevalent chronic illness.

We sought to refine an asthma symptom scale that RAND had previously developed to shorten it and validate it further, as well as reflect updated international expert definitions of asthma control. We conducted rigorous psychometric testing of new and adapted self-administered survey items in a sample of 2032 adults with asthma.

The reliability and preliminary validity of the resulting measure, henceforth referred to as the RAND Asthma Control Measure (RAND-ACM), matched or exceeded that of the original RAND measure and others in the literature. RAND-ACM scores for worse asthma control were significantly associated with worse asthma-related quality of life, increased asthma-related healthcare use, Hispanic ethnicity and lower educational level. Evidence for internal consistency was strong with a Cronbach's $\alpha$ of 0.84 . We also found adequate concordance between the RAND-ACM and the Global Initiative for Asthma categories of "uncontrolled", "partly controlled" and "controlled" asthma.

The RAND-ACM, a five-item self-reported asthma control survey measure, performs well in a large ethnically-diverse sample of US adults with asthma and provides a cost-free alternative to other asthma control measures currently available.

@ERSpublications

RAND-ACM, a five-item self-reported asthma control survey measure, performs well in a large sample of US asthmatics http://ow.ly/ygaZN

For editorial comments see page 1116.

Received: March 182014 | Accepted after revision: June 112014 | First published online: July 172014

Support statement: The content of this article is the sole responsibility of the authors. This work was supported by grant R01HL107312 from the US National Heart, Lung and Blood Institute.

Conflict of interest: Disclosures can be found alongside the online version of this article at erj.ersjournals.com

Copyright @ERS 2014 


\section{Introduction}

Assessing asthma control is not the only key aspect of successful management of this common chronic condition. Persons with asthma can better evaluate their treatment options when they are able to assess the control of their illness. In the clinical setting, evidence-based guidelines for care dictate how clinicians should titrate medication dosages and other treatments according to the patient's current level of asthma control. Researchers, healthcare organisations and others interested in the comparative effectiveness of alternative treatments and programmes also need reliable and valid measures of asthma control that can be used to assess the health of asthma populations.

The Global Initiative for Asthma (GINA) and US National Heart, Lung, and Blood Institute (NHLBI) guidelines for care define "asthma control" according to algorithms that include measures of patients" frequency of asthma symptoms, reported functional impairment and use of "rescue" medications or shortacting $\beta_{2}$-agonists that help relieve, but not prevent, asthma exacerbations [1,2]. Although the concept of asthma "control", as compared with "severity" and "symptoms", has received increased acceptance recently, the distinction among the definitions of these asthma morbidity domains is not always clear in clinical practice or the scientific literature [1-4]. Leaders in the asthma field participating in the 2010 NHLBI Asthma Outcomes Workshop reviewed some of these difficulties in assessing asthma control, and recommended several measures of asthma control in clinical, research and other settings [5].

RAND had previously developed an eight-item asthma symptom scale [6] with demonstrated internal consistency, reliability and validity in English-, Spanish- and Chinese-speaking parents of children with asthma [6,7], as well as adults with asthma [8]. Validity assessments indicated that the symptom scale discriminated among patients with intermittent and persistent asthma, was responsive to longitudinal changes in clinical states, and correlated significantly with asthma-related quality of life (QoL), pulmonary function, physician asthma ratings and healthcare use measures [6-8]. Because of these properties, the RAND asthma symptom scale, also referred to in the literature as the Lara Asthma Symptom Scale (LASS), has been designated as an "emergent" asthma control composite measure $[5,6]$.

As part of a NLHBI funded study to develop new measures for asthma-related QoL [9, 10], we had the opportunity to further refine and test the LASS [6] in a US, multiethnic sample. We set out to fine-tune the freely available scale to: 1) shorten it further; and 2) reflect updated expert definitions of asthma control that have been released since its original development [1-5]. The large sample size also gave us the opportunity to conduct more rigorous psychometric testing than the original evaluation, including preliminary examination of validity.

\section{Methods}

Participants

Harris Interactive (Rochester, NY, USA), a global interactive media and services company, recruited a national sample of US adults (aged $\geqslant 18$ years) with asthma $(n=2032)$ to undertake an Internet-based survey assessment $[9,10]$. All study procedures were approved by the RAND Corporation's institutional review board. Participants were eligible for the study if they answered "yes" to the following two questions: 1) "Has a doctor or healthcare professional ever told you that you have asthma?" and 2) "Do you still have asthma?" To ensure that we would have participants with a range of asthma morbidity, we also required that $90 \%$ of the sample answered "yes" to the following question: "During the past 12 months have you had an episode of asthma or an asthma attack?" As described elsewhere [10], the sampling approach included some oversampling of Hispanic, African-American and Asian groups, and inclusion of individuals in each of four age groups (18-24 years, 35-49 years, 50-64 years and $\geqslant 65$ years). Table 1 describes the demographic characteristics and asthma-related healthcare utilisation of the participants.

\section{Survey measures}

Participants completed a self-administered Internet-based survey including items modified from the original RAND measure (LASS) [6], other validated asthma control [11] and asthma-related QoL measures $[10,12,13]$, as well as other survey items for constructs that were hypothesised to be associated with level of asthma control.

Items tested for the new control measure

We considered a total of 10 items for the new control measure. Eight of these were based on the original LASS [6]: two were retained unchanged from the LASS (perceived severity and a free response indicator of the number of asthma attacks in the past month), six LASS items (cough, wheezing, chest pain, asthma attack frequency, awakened at night and shortness of breath) were included with revised response options as shown in table 2. The two new items (interference of normal activities and frequency of rescue inhaler use) 
TABLE 1 Demographic characteristics of the sample of 2032 adults with asthma

$\begin{array}{lc}\text { Age years } & 43 \pm 15 \\ \text { Females } & 1219(60) \\ \text { Ethnicity/race } & 282(14) \\ \quad \text { Hispanic } & 221(11) \\ \text { Asian } & 381(19) \\ \text { African-American } & 1148(56) \\ \text { Non-Hispanic white } & \\ \text { Region of the USA } & 478(24) \\ \text { East } & 432(21) \\ \text { Midwest } & 578(28) \\ \text { South } & 544(27) \\ \text { West } & 353(17) \\ \text { Education } & 743(36) \\ \text { High school or below } & 490(24) \\ \text { Some college } & 446(22) \\ \text { College graduate } & \end{array}$

Data are presented as mean \pm SD or $\mathrm{n}(\%)$.

were developed to more closely reflect the control categories defined by GINA [1] and NHLBI expert panel report 3 (table 2) [2]. Other than the free response indicator of number of asthma attacks, all but one item was assessed with a five-category response scale, with higher scores indicating worse control. Activity interference had a four-category response scale.

\section{Other control and asthma-related QoL survey measures included}

The Asthma Control Test (ACT) is a widely used measure for asthma control consisting of five items, each using a five-point Likert response scale, with a total score range from 5 (poor control) to 25 (good control), and score categories of 5-15 (poorly controlled), 16-19 (somewhat controlled) and 20-25 (well controlled) as established in the original validation studies [11]. Among other measures for asthma control reported in the literature, we chose the ACT for comparative purposes because it does not require results of pulmonary function tests (forced expiratory volume in $1 \mathrm{~s}$ ) in the categorisation of levels of asthma control [5].

The AQLQ-Marks (Marks Asthma Quality of Life Questionnaire) [12,13] is a 20-item measure that has been used in a number of studies over the years within and outside the USA, and the RAND-IAQL-12 (RAND Negative Impact of Asthma on Quality of Life 12-item Short Form) is a newly developed measure representing a broad content range of asthma impact [10]. The RAND-IAQL-12 items have excellent internal consistency (marginal reliability $=0.93$ ) [9] and a recent study reports preliminary validity evidence for the measure [10].

\section{Other survey items included}

These included indicators for demographic characteristics (sex, ethnicity, age and education) and asthmarelated healthcare utilisation (free response indicators of whether and how many times the participant had been hospitalised for asthma during the past 12 months; had been seen in an emergency department for asthma during the past 12 months; and whether and how frequently, on average, the participant had taken controller medications in the past 4 weeks).

\section{Survey data analyses}

The objectives of the analyses were as follows. 1) Revise the original RAND measure by evaluating the modified set of 10 fielded items using factor analyses to examine dimensionality and arrive at a final set of items to reliably measure asthma control, henceforth referred to as the RAND Asthma Control Measure (RAND-ACM). 2) Conduct preliminary validation of the RAND-ACM, involving the relationship of the RAND-ACM with the ACT, the selected measures of asthma-specific QoL, and the demographic and asthma-related healthcare utilisation indicators. 3) Evaluate the concordance of the RAND-ACM with the GINA and NHLBI control categorisations, using estimates of the area under the curve (AUC) as well as sensitivity, specificity and associated $\kappa$ coefficients. The concordance analyses were repeated using the ACT in place of the RAND-ACM to provide a basis for comparison. 
TABLE 2 RAND-ACM item response frequencies and factor loadings

...how often did you have an asthma attack?

Not at all

Once or twice a week

3-6 times a week

Once a day

More than once a day

...how often have you been awakened at night because of your asthma symptoms?

Never

1 or 2 times during the past 4 weeks

3 or 4 times during the past 4 weeks

1 or more times a week but not every night

Every night

...how much did your asthma interfere with your normal activities?

Not at all

A little

A moderate amount

A lot

...how often have you used a rescue inhaler that gives quick relief from asthma symptoms?

Never

1 time per week

2 or more times per week but not daily

Daily

Several times a day, most days

...how often did you have shortness of breath?

Not at all

Once or twice a week

3-6 times a week

Once a day

More than once a day
$882(43.60)$
$799(39.50)$
$207(10.23)$
$69(3.41)$
$66(3.26)$
$817(40.23)$
$571(28.11)$
$326(16.05)$
$238(11.72)$
$79(3.89)$

518 (25.52)

926 (45.62)

$461(22.71)$

$125(6.16)$

495 (24.42)

$510(25.16)$

$600(29.60)$

$279(13.76)$

$143(7.05)$

279 (13.77)

935 (46.15)

$406(20.04)$

$160(7.90)$

$246(12.14)$

RAND-ACM: RAND Asthma Control Measure. " : all items began with the phrase "During the last 4 weeks," and were self-administered in the order shown. For comparison to the original RAND Lara Asthma Symptom Scale items please see LARA et al. [6].

\section{Factor analyses and selection of items for RAND-ACM}

We first conducted an exploratory factor analysis to ensure the unidimensionality of the 10 asthma control test items. This was followed by a series of one-factor confirmatory factor analyses (CFA). We considered the item loadings and modification indices from the CFAs to arrive at a shortened instrument with adequate content breadth and reliability. A random subset of the data $(n=532)$ was set aside to confirm the fit of the final model; thus, all developmental factor analyses were conducted with $n=1500$ observations. Factor analyses were conducted using the computer program Mplus [14], and the mean and variance adjusted weighted least squares algorithm that is appropriate for categorical response items. Model fit was evaluated with commonly used model fit indices (root mean square error of approximation (RMSEA) $\leqslant 0.08$; Tucker-Lewis index (TLI) $\geqslant 0.95$; comparative fit index $(\mathrm{CFI}) \geqslant 0.95)$.

\section{Preliminary validity analyses}

We examined correlations of the RAND-ACM with the ACT, the RAND-IAQL-12 and the AQLQ-Marks, hypothesising that the RAND-ACM would be negatively correlated with the ACT (because the RAND-ACM and ACT have opposite directionality) and positively correlated with the two measures of asthma-specific QoL. We also examined mean differences in RAND-ACM scores according to the demographic and asthmarelated healthcare utilisation indicators. For these constructs, we hypothesised that RAND-ACM means would be higher (worse control) for individuals with lower education, females, younger adults and individuals with higher asthma-related healthcare use (more emergency room visits, hospital stays and controller medication use) and/or self-identified as Hispanic or African-American [15].

\section{Concordance with GINA and NHLBI asthma control classifications}

The three control categorisations of GINA (uncontrolled, partly controlled and controlled) [1] and the NHLBI (very poorly controlled, not well controlled and well controlled) [2] were assigned based on 
responses to eight of the 10 modified LASS items querying symptoms (five items), awakening at night, normal activity interference and rescue inhaler use.

As a first step we examined mean differences in RAND-ACM scores according to the GINA and NHLBI control classifications, hypothesising that the lowest means would correspond to the highest control categories and the highest means would correspond to the more poorly controlled classifications.

Then we conducted logistic regression analyses using the RAND-ACM (or ACT) to predict GINA and NHLBI control classifications and obtain estimates of the AUC associated with discrimination of each outcome. Next we evaluated the concordance, including sensitivity, specificity and $\kappa$ coefficients, of a series of RAND-ACM (or ACT) cut-off points (e.g. scores $>5,>6$ and $>7$ ) with GINA and NHLBI category boundaries. Although we examined all possible cut-off points, we tabulated results for the RAND-ACM and ACT cut-off points that yielded the three highest $\kappa$ values. Finally, we generated graphs to demonstrate concordance of the RAND-ACM (and ACT) with the GINA and NHLBI classifications. Although there are multiple ways to determine cut-off points for this purpose, we chose the RAND-ACM and ACT cut-off points that yielded the highest $\kappa$ coefficients. To facilitate comparisons with existing literature, the graphs also included concordance based on established cut-off points for the ACT [11].

\section{Results}

\section{Factor analyses and reliability of RAND-ACM}

Exploratory factor analysis results for the 10 control items supported a single factor solution. The first factor accounted for $63 \%$ of the total variance, and all item loadings were strong, positive and significant (range 0.62-0.90). Results from a one-factor CFA of the 10 items indicated some misfit (Chi-squared 1267.1, degrees of freedom (df) 35; CFI 0.950; TLI 0.935; RMSEA 0.153). Based on the modification indices from this solution, and in consideration of psychometric performance as well as preferences with respect to clinical judgment face validity and ease of later translation to Spanish, we identified five items for removal (cough, wheeze, chest pain, actual number of attacks and severity). Fit of the CFA model for the final fiveitem set was excellent, providing strong evidence of unidimensionality (Chi-squared 11.8, df 5; CFI 0.999; TLI 0.998; RMSEA 0.030), which was confirmed in the set-aside subset of the data (Chi-squared 5.2, df 5; CFI 1.0; TLI 1.0; RMSEA 0.008). The final five items included in the measure are shown in table 2 with their respective response frequencies and factor loadings. The RAND-ACM score, calculated as the sum of responses to the five items, ranges from five to 24 , with a mean \pm SD of $11.16 \pm 4.24$. A high score reflects worse control. Cronbach's $\alpha$ for the five-item measure was 0.84 .

\section{Preliminary validity of the RAND-ACM}

Support for the convergent validity of the RAND-ACM was evidenced by its large significant correlations with the ACT (-0.85), AQLQ-Marks (0.72) and RAND-IAQL-12 (0.75). Furthermore, as hypothesised and shown in table 3, RAND-ACM means differed significantly according to indicators of the frequency of asthma attacks, overnight hospital stays and emergency department visits in the past year, the extent of average daily controller medication use in the past 4 weeks, self-rated severity over the past 4 weeks, and levels of control based on both GINA and NHLBI guidelines, providing evidence of validity. Although the pattern of RAND-ACM mean scores according to demographic groups did not support all our hypotheses (Hispanic subjects had worse asthma control scores than non-Hispanic white subjects, but AfricanAmericans did not), we did observe improved control with increasing education as expected. There were no differences in control according to sex and, although the overall difference test for age was significant at $\mathrm{p}<0.05$, none of the pairwise group mean comparisons were significant.

\section{Concordance with GINA and NHLBI asthma control classifications}

A final set of evaluations used logistic regression analysis to compare the performance of the RAND-ACM to the ACT in predicting GINA and NHLBI control categories. As shown in table 4, the large AUCs indicated that both the RAND-ACM and the ACT were significantly associated with the control categories. However, the RAND-ACM performed somewhat better in distinguishing between both the GINA- and the NHLBI-defined control categories (RAND-ACM AUC range 0.845-0.968, ACT AUC range 0.767-0.943; all Chi-squared tests for differences in AUC between RAND-ACM and ACT were significant at $\mathrm{p}<0.0001$ ).

Table 4 also displays the sensitivity, specificity and associated $\kappa$ statistic for agreement corresponding to several cut-off points on each control measure for distinguishing between both the GINA and NHLBI defined control categories. The cut-off points associated with the three highest $\kappa$ coefficients are presented for each comparison. The sensitivity and specificity values tend to be more favourable for the RAND-ACM as compared with the ACT, and the RAND-ACM also achieves higher levels of agreement according to the $\kappa$ statistic, although these trends were not formally tested. It is also noteworthy that both control measures are 
TABLE 3 Differences in RAND-ACM means according to select groups

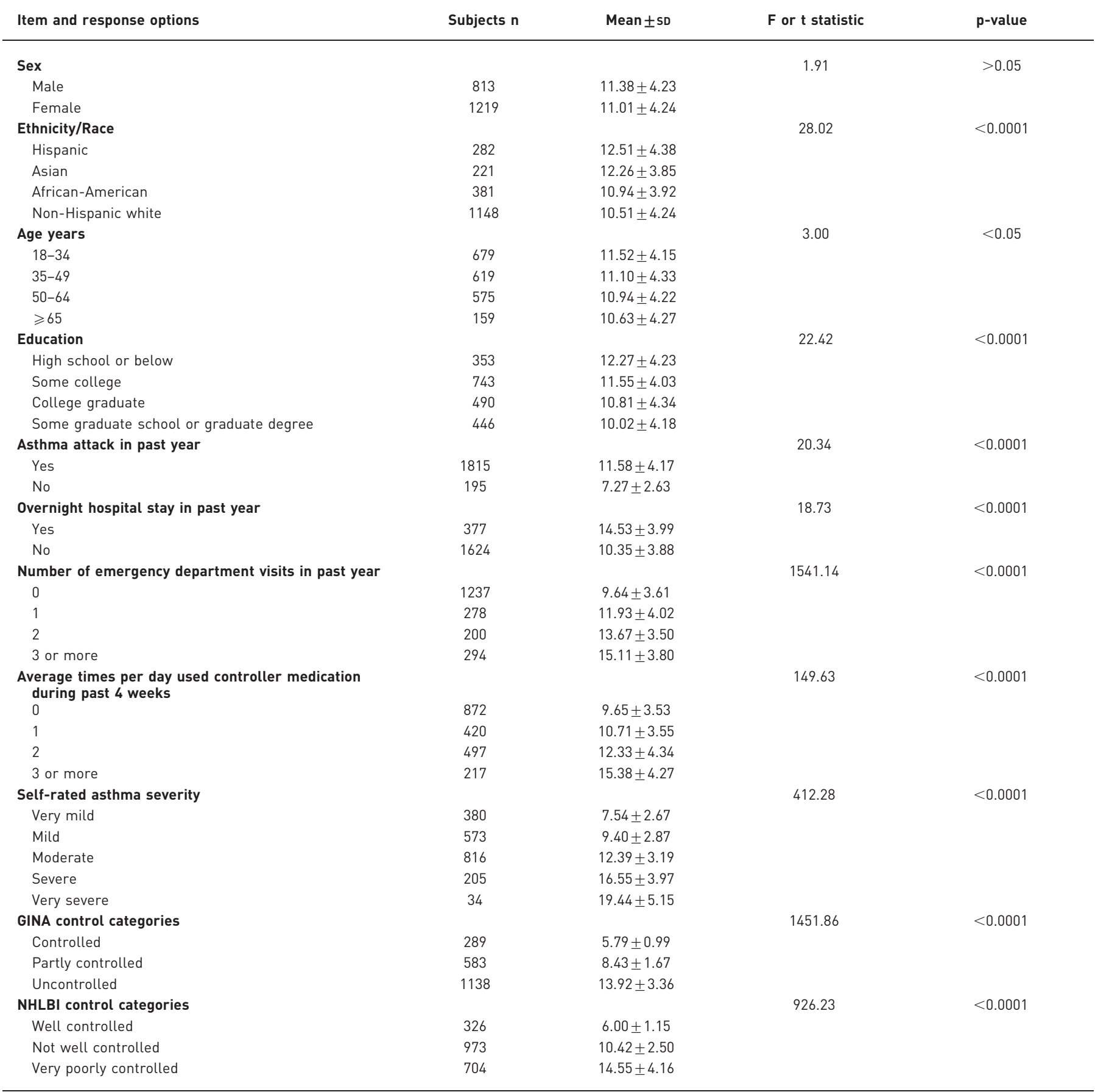

RAND-ACM: RAND Asthma Control Measure; GINA: Global Initiative for Asthma; NHLBI: National Heart, Lung, and Blood Institute.

better at distinguishing the highest (most controlled) respondents from those with moderate or poor control, and this trend is apparent for both the GINA and NHLBI categorisations.

Using the table 4 cut-off points associated with the highest $\kappa$ values, figures 1 and 2 demonstrate the concordance of the RAND-ACM and the ACT with the GINA and NHLBI control classifications. Specifically, figure 1 shows the proportion of our sample that is classified into each NHLBI control category by the RAND-ACM (well controlled, 5-7; not well controlled, 8-12; very poorly controlled, 13-24), the ACT based on our empirical cut-off points from the data (well controlled, 24-25; not well controlled, 17-23; very poorly controlled, 5-16) and the ACT using previously established cut-off points (well 


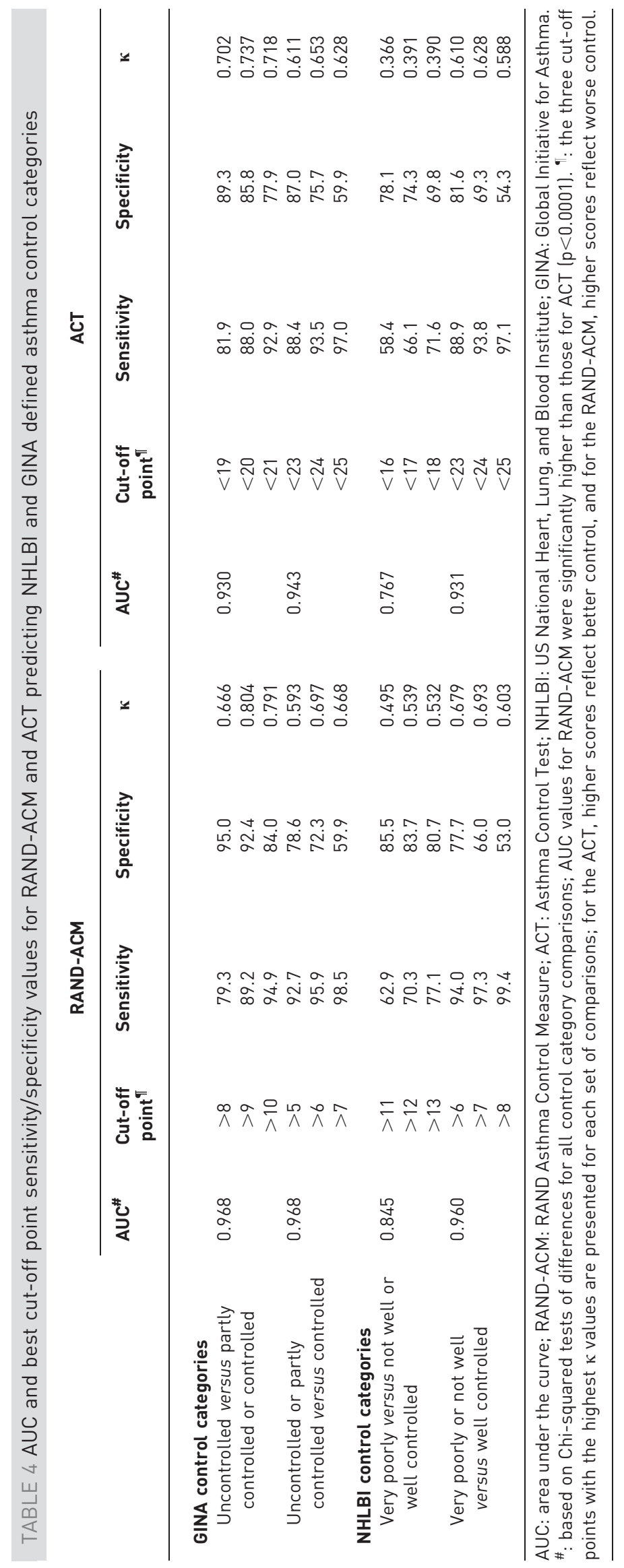


Very poorly controlled $\square$ Not well controlled $\square$ Well controlled

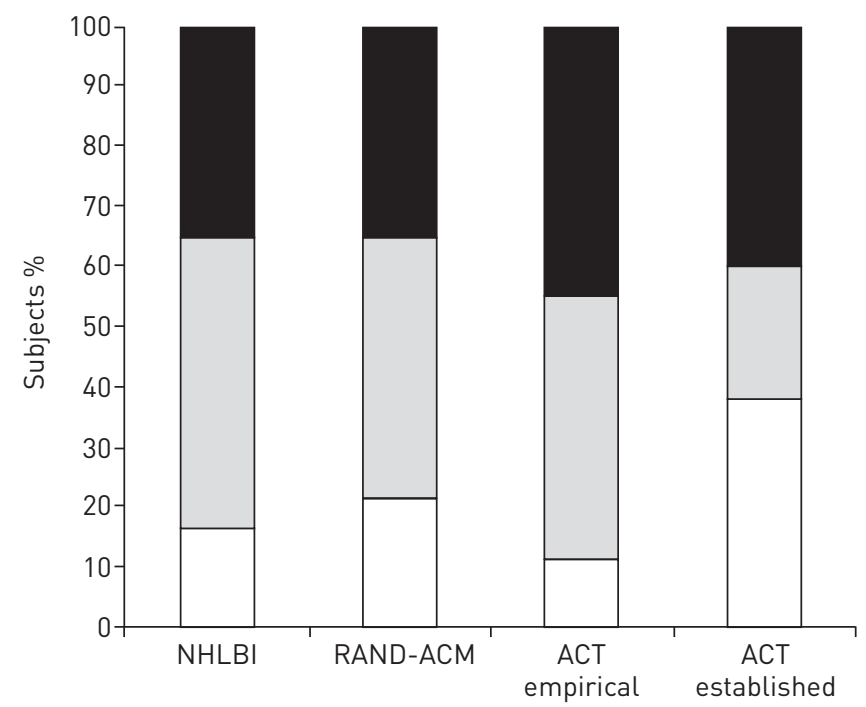

Measure

FIGURE 1 Concordance of RAND Asthma Control Measure (RAND-ACM) and Asthma Control Test (ACT) with US National Heart, Lung, and Blood Institute (NHLBI) control categorisation. For the ACT, empirical cut-off points were derived from the data, whereas established cut-off points were cut-off points that had previously been used with the ACT.

controlled, 20-25; not well controlled, 16-19; very poorly controlled, 5-15). Figure 2 shows comparable proportions for classification into each GINA control category. Examination of these figures reveals that the RAND-ACM results in higher concordance than the ACT using either the empirical or established ACT cutoff points with both the GINA and NHLBI control classifications.

\section{Discussion}

Our results show the strong psychometric properties of the RAND-ACM, a five-item survey measure of asthma control that was refined and adapted from a previous asthma symptom measure and is available to researchers and clinicians at no cost. The preliminary validity results of the RAND-ACM presented here

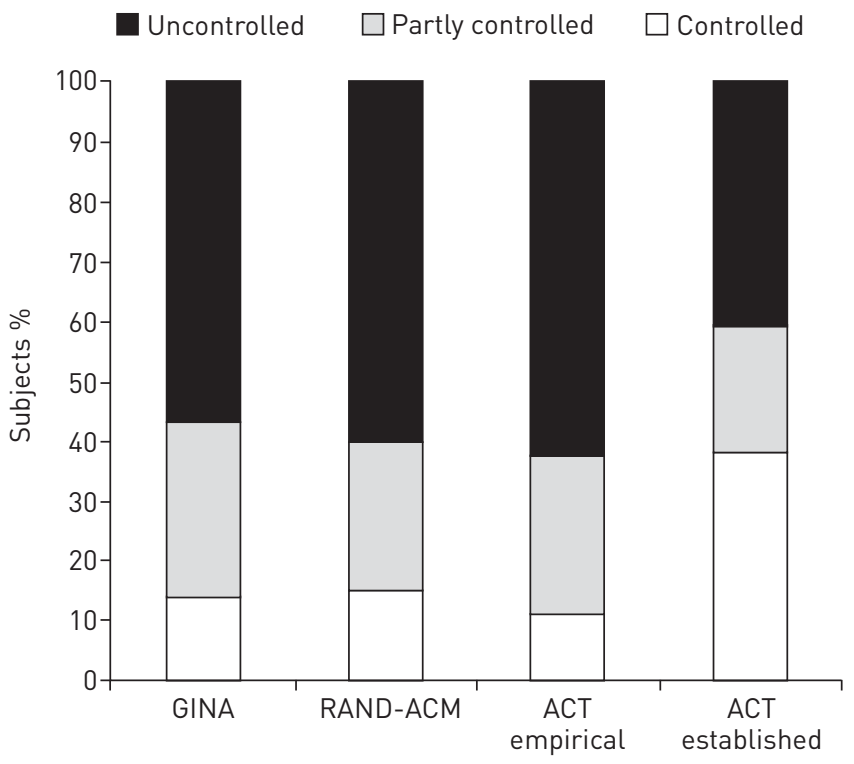

Measure

FIGURE 2 Concordance of RAND Asthma Control Measure (RAND-ACM) and Asthma Control Test (ACT) with Global Initiative for Asthma (GINA) control categorisation. For the ACT, empirical cut-off points were derived from the data, whereas established cut-off points were cut-off points that had previously been used with the ACT. 
match or exceed the original measures and other asthma control measures described in the literature [5]. Specifically, RAND-ACM scores for worse asthma control were significantly associated with worse asthmarelated QoL, increased asthma-related healthcare use, Hispanic ethnicity and lower educational level. In terms of reliability, the RAND-ACM has strong evidence of internal consistency.

In the absence of a "gold standard" for asthma control, we evaluated the concordance of asthma control categories derived from RAND-ACM scores with those outlined by the most recent GINA [1] and NHLBI [2] guidelines for asthma. Overall, we found adequate concordance between the RAND-ACM and the GINA categorisation of the study population into "uncontrolled," "partly controlled," and "controlled" asthma categories, and with the NHLBI categories of "very poorly controlled," "not well controlled," and "well controlled" asthma.

For the concordance analyses, we chose RAND-ACM cut-off points that maximised the $\kappa$ between the RAND-ACM and the GINA and NHLBI classifications. However, the ideal cut-off score may vary depending on the intended use of the score, the population being classified and the personal preferences of the researcher. Our intent in presenting the sensitivity, specificity and $\kappa$ associated with multiple cut-off scores for the RAND-ACM was to provide readers with sufficient information to make their own choice as to optimal cut-off score for a particular application.

Our study also demonstrated some other interesting findings. The factor analyses supported the probable one-dimensional nature of the "asthma symptoms" and "asthma control" items from the patient's perspective since we did not find any justification for the need for "subscales." Although a head-to-head comparison of the RAND-ACM with the ACT was not a primary objective of the analyses, we found that the concordance between the ACT categories of control and those of GINA and the NHLBI, respectively, were much better when we used cut-off points for the ACT that were empirically driven by our own sample, instead of those established in previous studies of the ACT. Others have described how the previously established cut-off points for the ACT may over- or underestimate asthma control categories as compared with GINA [16]. The lack of agreement among different measures of control has also been documented in the literature $[17,18]$.

Our findings need to be interpreted in light of the limitations of our study. Our study was cross-sectional so we could not assess longitudinal test-retest reliability for the RAND-ACM. Because our sample was Internet and not clinic based, we could not assess the responsiveness of the RAND-ACM to treatment or any associations with changes in pulmonary function (i.e. before/after a hospitalisation or emergency room visit). Unlike the original LASS measure, our initial testing of the RAND-ACM was conducted in Englishspeaking patients only. However, we believe that the strong performance of the RAND-ACM in the current sample would probably be replicable in a Spanish-speaking sample, given that the original LASS measure had equivalent properties in both English and Spanish, and the modifications to the LASS in the creation of the RAND-ACM were not major. Likewise, since the original LASS is responsive to change and is related to changes in pulmonary function $[6,8]$, one could speculate that the RAND-ACM would also reflect changes in clinical assessments. However, more research is needed in well-defined clinical samples to test these hypotheses. Finally our results are based on data from a relatively well-educated adult sample. Thus, further evaluation of the RAND-ACM is needed in a general adult population as well as in children.

\section{Summary}

We have confirmed the good performance of the RAND-ACM in an ethnically diverse cross-sectional sample of adults with asthma. The RAND-ACM was modified from the previously validated LASS to reflect an increased emphasis on asthma control versus asthma symptoms or severity in expert-based international definitions of asthma morbidity. The RAND-ACM provides a cost-free alternative to other asthma control measures currently described in the literature. Additional research is necessary to understand the degree to which these favourable results for the RAND-ACM in an English-speaking, Internet-based sample in the USA are applicable to other populations.

\section{Acknowledgements}

The authors would like to thank all persons with asthma who participated in the study. Marielena Lara would like to thank her husband, Richard Greenberg, and their daughter, Serena Michelle Lara-Greenberg, for their unwavering support.

\section{References}

1 Global Initiative for Asthma. Global Strategy for Asthma Management and Prevention. www.ginasthma.org/local/ uploads/files/GINA_Report_2014_Jun11.pdf Date last updated: June, 2014. Date last accessed: March 17, 2014.

2 National Heart, Lung, and Blood Institute, National Asthma Education and Prevention Program. Expert Panel Report 3: Guidelines for the Diagnosis and Management of Asthma. Bethesda, National Heart, Lung, and Blood Institute, 2007. 
3 Taylor DR, Bateman ED, Boulet LP, et al. A new perspective on concepts of asthma severity and control. Eur Respir J 2008; 32: 545-554.

4 Reddel HK, Taylor DR, Bateman ED, et al. An official American Thoracic Society/European Respiratory Society statement: asthma control and exacerbations: standardizing endpoints for clinical asthma trials and clinical practice. Am J Respir Crit Care Med 2009; 180: 59-99.

5 Cloutier MM, Schatz M, Castro M, et al. Asthma outcomes: composite scores of asthma control. J Allergy Clin Immunol 2012; 129: Suppl. 3, S24-S33.

6 Lara M, Sherbourne C, Duan N, et al. An English and Spanish pediatric asthma symptom scale. Med Care 2000; 38: 342-350.

7 Chen CY, Lo LH. Reliability and validity of a Chinese version of the pediatric asthma symptoms scale. J Nurs Res 2007; 15: 99-105.

8 Wood PR, Smith B, O'Donnell L, et al. Quantifying asthma symptoms in adults: the Lara Asthma Symptom Scale. J Allergy Clin Immunol 2007; 120: 1368-1372.

9 Stucky BD, Edelen MO, Sherbourne CD, et al. Developing an item bank and short forms that assess the impact of asthma on quality of life. Respir Med 2014; 108: 252-263.

10 Sherbourne CD, Stucky BD, Edelen MO, et al. Assessing the validity of the RAND Negative Impact of Asthma on Quality of Life Short Forms. J Allergy Clin Immunol 2014 [In press DOI: 10.1016/j.jaci.2014.03.002].

11 Schatz M, Sorkness CA, Li JT, et al. Asthma Control Test: reliability, validity, and responsiveness in patients not previously followed by asthma specialists. J Allergy Clin Immunol 2006; 117: 549-556.

12 Marks GB, Dunn SM, Woolcock AJ. A scale for the measurement of quality of life in adults with asthma. J Clin Epidemiol 1992; 45: 461-472.

13 Marks GB, Dunn SM, Woolcock AJ. An evaluation of an asthma quality of life questionnaire as a measure of change in adults with asthma. J Clin Epidemiol 1993; 46: 1103-1111.

14 Muthén LK, Muthén BO. Mplus: Statistical Analysis with Latent Variables. User's Guide. 6th Edn. Los Angeles, Muthén \& Muthén, 2010.

15 Akinbami LJ, Moorman JE, Liu X. Asthma prevalence, health care use, and mortality: United States, 2005-2009. Natl Health Stat Report 2011; 32: 1-14.

16 Koolen BB, Pijnenburg MWH, Brackel HJK, et al. Comparing Global Initiative for Asthma (GINA) criteria with the Childhood Asthma Control Test (C-ACT) and Asthma Control Test (ACT). Eur Respir J 2011; 38: 561-566.

17 Green RJ, Klein M, Becker P, et al. Disagreement among common measures of asthma control in children. Chest 2013; 143: 117-122.

18 Vermeulen F, de Meulder I, Paesmans M, et al. Asthma control measurement using five different questionnaires: a prospective study. Respir Med 2013; 107: 1314-1321. 